IGCS19-0099

\section{STARTING A HIPEC PROGRAMME IN A LOW RESOURCE SETTING}

${ }^{1} \mathrm{~A}$ Peedicayil ${ }^{*},{ }^{2} \mathrm{R}$ Mittal, ${ }^{3} \mathrm{~A}$ Joel, ${ }^{4} \mathrm{E}$ Rai, ${ }^{1} \mathrm{~A}$ Sebastian, ${ }^{1} \mathrm{~V}$ Thomas, ${ }^{1} \mathrm{~A}$ Thomas, ${ }^{1} \mathrm{R}$ Chandy, ${ }^{5} \mathrm{~K}$ Subramani. ${ }^{1}$ Christian Medical College, Gynaecologic Oncology, Vellore, India; ${ }^{2}$ Christian Medical College, Colorectal Surgery, Vellore, India; ${ }^{3}$ Christian Medical College, Medical Oncology, Vellore, India; ${ }^{4}$ Christian Medical College, Anaesthesia, Vellore, India; ${ }^{5}$ Christian Medical College, Critical Care, Vellore, India

10.1136/ijgc-2019-IGCS.320

Objectives Hyperthermic IntraPeritoeneal Chemotherapy (HIPEC) after maximal cytroreduction is a promising modality of treating women with ovarian cancer. In order to determine the feasibility of setting up a HIPEC programme in India, we document our initial experience.

Methods Ethics Committee clearance was obtained to start the programme. The electronic medical records of all patients who underwent HIPEC in our department was reviewed.

Results A total of 14 patients underwent HIPEC in the first 2 years: one primary, 6 interval and 7 recurrent cytoreductions.

The women had a mean age of 46.9 years (36 to 62), median performance score of 1 (o to 2 ) and a median peritoneal carcinomatosis index (PCI) of 10 (2 to 25).

The histology was serous in 9 , mucinous in 4 and endometrioid in one.

Four patients had bowel resection of whom 2 had an end ileostomy and one had an end colostomy. The median duration of surgery was 9 hours (5 to 10) and the median completeness of cytoreduction score was 1 (0 to 2). The drugs used in HIPEC were Cisplatin and Oxaliplatin. The median duration of hospital stay was 9 days (6 to 21).

Two patients were readmitted to hospital and 3 patients had re-laparotomy. The main complications were venous thromboembolism in one, bleeding in one and wound dehiscence in one.

Conclusions Cytoreductive surgery with HIPEC is feasible in a low resource setting with acceptable morbidity where the main limitations are non-availability of operating time and patient's ability to pay for treatment.

\section{IGCS19-0574}

\section{MAINTENANCE OLAPARIB FOR BRCA-MUTATED OVARIAN CANCER (OC) PATIENTS IN 1ST LINE AND PLATINUM-SENSITIVE RELAPSED (PSR) SETTINGS: MAXIMIZING TREATMENT OPPORTUNITIES}

${ }^{1}$ A Poveda*, ${ }^{2}$ A Sackeyfio, ${ }^{3} \mathrm{M}$ Friedlander. ${ }^{1}$ Initia Oncology, Department of Gynecologic Oncology, Valencia, Spain; '2AstraZeneca, Health Economics and Payer Analytics, Cambridge, UK; ${ }^{3}$ Prince of Wales Hospital, University of New South Wales Clinical School, Randwick, Australia

\subsection{6/ijgc-2019-IGCS.321}

Objectives In Phase III randomized trials, maintenance therapy with the PARP inhibitor olaparib demonstrated a significant benefit versus placebo in BRCA-mutated advanced OC patients who had a complete/partial response to platinum-based chemotherapy (PBC) in newly diagnosed (SOLO1; NCT01844986) and PSR (SOLO2/ENGOT-Ov21; NCT01874353) settings. We investigate missed opportunities for maintenance olaparib in relapsed settings.

Abstract 321 Table 1
\begin{tabular}{lccc}
\hline Patient setting & Cohort, $\mathbf{n}$ & Calculation & $\begin{array}{c}\text { Population eligible for } \\
\text { olaparib, } \mathbf{n}(\%)\end{array}$ \\
\hline Newly diagnosed & 1000 & 1000 & $844(84)$ \\
BRCAm & & $\times 0.75$ (first-line response rate) \\
& \multicolumn{3}{c}{1.12 (BRCA response rate factor) } \\
\hline PSR OC & 1000 & $\times 0.75$ (first-line response rate) \\
BRCAm & \multicolumn{3}{c}{$439(44)$} \\
& $\times 1.12$ (BRCA response rate factor) \\
& $\times 0.806$ (platinum sensitivity) \\
& $\times 0.646$ (second-line response rate) \\
\hline
\end{tabular}

Methods Published response rates to PBC in newly diagnosed BRCA-mutated advanced OC are scarce. These are estimated by applying a BRCA response rate factor determined from a population-based study (Alsop et al) to expected $\mathrm{PBC}$ response rates in newly diagnosed high grade serous OC (75\%). For BRCA-mutated PSR OC, response to second-line PBC was 64.6\% (Alsop et al). Platinum sensitivity for second-line PBC eligibility was determined from placebo patients in SOLO1 remaining progression free after 6 months (80.6\%).

Results In newly diagnosed and PSR settings, predicted proportions of eligible patients for olaparib are $84 \%$ and $44 \%$, respectively (table 1). Missed treatment opportunities in PSR settings are likely due to platinum resistance and non-response to second-line PBC. Approximately $48 \%$ of patients could miss the opportunity to benefit from PARP inhibitor maintenance if untreated in the first line.

Conclusions Earlier olaparib therapy provides the chance of long-term remission and prevents patients missing opportunities for second-line PARP inhibitor maintenance due to platinum resistance or non-response to PBC. Disease burden associated with multiple chemotherapy lines in advanced settings is also reduced or delayed.

\section{IGCS19-0595}

\section{THE USE OF CURETTAGE IN THE MANAGEMENT OF DIAPHRAGMATIC INVOLVEMENT IN PATIENTS WITH PRIMARY ADVANCED-STAGE OVARIAN OR PERITONEAL CANCER}

R Puente*, E Schneider. Austral University, Obstetrics and Gynecology Institute. Gynecologic Oncology Section, Valdivia, Chile

\subsection{6/ijgc-2019-IGCS.322}

Objectives To know the usefulness of diaphragm curettage to remove all metastasis in patients with primary advanced-stage ovarian or peritoneal cancer, with diaphragmatic involvement. Methods In 16 consecutive patients with advanced primary epithelial ovarian or peritoneal cancer with diaphragmatic involvement we performed diaphragm curettage with a big sharp curette (Aesculap N 16) as a part of cytoreductive surgery, after liver mobilization. We used narrow curettes in areas with difficult access. The procedure had limited bleeding controlled by coagulation and hot compress.

Results In all 16 patients, the curettage removed completely the tumor implants, in one or both diaphragms, without residual disease. In the postoperative time, 3 patients had basal pleural effusion, reasorbed spontaneously.

Conclusions Diaphragmatic curettage is a safe and effective procedure to treat the diaphragm involvement in cytoreductive 
surgery of ovarian or peritoneal carcinoma, decreasing the morbidity of the surgery.

\section{IGCS19-0098}

\section{MRNA AND PROTEIN EXPRESSION OF E-CADHERIN AND VIMENTIN AND P53 IMMUNOHISTOCHEMISTRY IN EPITHELIAL OVARIAN CANCER}

${ }^{1} S$ Rajaram*, ${ }^{1} S$ Chaudhary, ${ }^{2} \mathrm{BD}$ Banerjee, ${ }^{3} \mathrm{VK}$ Arora, ${ }^{1} \mathrm{~B}$ Gupta, ${ }^{4} \mathrm{PK}$ Garg, ${ }^{1} \mathrm{~S}$ Jain. ${ }^{1}$ University College of Medical Sciences and Guru Teg Bahadur Hospital, Department of Obstetrics and Gynecology, Delhi, India; ${ }^{2}$ University College of Medical Sciences and Guru Teg Bahadur Hospital, Department of Biochemistry, Delhi, India; ${ }^{3}$ University College of Medical Sciences and Guru Teg Bahadur Hospital, Department of Pathology, Delhi, India; ${ }^{4}$ All India Institute of Medical Sciences, Surgical Oncology, Rishikesh, India

\subsection{6/ijgc-2019-IGCS.323}

Objectives This study was designed to correlate expression of epithelial mesenchymal transition (EMT) pathway markers i.e., E-cadherin and Vimentin with surgicopathological extent of EOC and to type the tumour using p53 immunohistochemistry staining.

Methods Women with malignant and benign epithelial ovarian tumours were studied. Sample size was calculated with $80 \%$ power and 5\% level of significance ;22 cases (EOC) and 22 controls (benign ovarian tumour) were recruited. m-RNA and protein expression of E-cadherin and vimentin was done by real time PCR and IHC staining and p53 by IHC. Peritoneal extent of disease was calculated by peritoneal carcinomatosis index (PCI) and tumour resection by completeness of cytoreductive score (CCS) and correlations derived.

Results In advanced EOC, positive correlation was found between PCI and CCS with correlation coefficient of 0.495 , $\mathrm{p}$-value $<0.0193$. When PCI less than $10(\mathrm{n}=10)$, CCS0 was achieved. m-RNA expression of E-cadherin was 2.126 times downregulated and of vimentin 2.733 times upregulated in malignant vs. benign tumours. Protein expression of E-cadherin was high in benign vs. malignant $\operatorname{EOC~}(p=0.387)$ and vimentin protein expression was overexpressed in EOC $(p=0.007)$. No correlation was obtained between EMT markers and metastatic deposits, lymph node or bowel involvement. p53 was expressed in 90.9\% $(n=10)$ high grade serous carcinoma and none in low grade serous carcinoma.

Conclusions Expression of E-cadherin decreased and Vimentin increased in EOC which is in synchrony with EMT pathway, however larger studies are needed to derive an association between these markers and extent of disease.

\section{IGCS19-0296}

\section{MUTATIONAL PROFILING OF BRAZILIAN MUCINOUS ADENOCARCINOMA OF THE OVARY}

${ }^{1} \mathrm{~L}$ Ferrante, ${ }^{2} \mathrm{~N}$ Campanella, ${ }^{3} \mathrm{R}$ Dufloth Mucha, ${ }^{4} \mathrm{C}$ Andrade, ${ }^{4} \mathrm{G}$ Cintra Fontes, ${ }^{4} \mathrm{M}$ Vieira $\mathrm{A}$ ${ }^{2} \mathrm{R}$ Reis Manuel, ${ }^{4} \mathrm{R}$ Reis*. ${ }^{1}$ Faculdade de Ciências da Saúde de Barretos Dr Paulo Prata Medical student, Barretos, Brazili; ${ }^{2}$ Fundação PIO XII, Centro de Pesquisa em Oncologia Molecular, Barretos, Brazil; ${ }^{3}$ Fundação PIO XII, Pathology Department, Barretos, Brazil; ${ }^{4}$ Fundação PIO XII, Gynecologic Oncology Department, Barretos, Brazil

10.1136/ijgc-2019-IGCS.324
Objectives To investigate the mutational profiling associated with Brazilian mucinous adenocarcinoma of ovary.

Methods We included 47 patients from Barretos Cancer Hospital, from 2009 and 2015. The mutation profile of a panel of hotspot regions of 15 cancer drivers (AKT1, BRAF, EGFR, ERBB2, FOXL2, GNA11, GNAQ, KIT, KRAS, MET, NRAS, PDGFRA, PIK3CA, TP53) was performed by NGS using the Illumina TruSight Tumor 15 panel on MiSeq instrument (Illumina, USA) in a subset of cases. Hotspots regions of the KRAS (codons 12 and 13) were screened by PCR followed by direct Sanger sequencing. Finally, HER2 amplification was evaluated by immunohistochemistry (IHC) and in situ hybridization (FISH).

Results HER2 IHC was performed in 27 samples that showed absence of staining in 25 (92.6\%). Two samples $(7.4 \%)$ showed inconclusive IHC reaction, and FISH analysis showed HER2 amplification in both cases. Clinically, both cases had pseudomixoma peritonei and 1 had disease recurrence. For KRAS Sanger sequencing, conclusive results were obtained in 25 cases due to DNA quality issues. KRAS mutations were found in 10 samples (40\%), and were associated with no recurrence $(p=0.036)$. The TruSight Tumor 15 panel was possible only in 16 samples. All samples showed at least one mutation in one gene and the most mutated genes were: TP53 and KRAS (10 mutations each), BRAF (4) and HER2 and PIK3CA (1).

Conclusions We identified potential therapeutic targets in Brazilian mucinous adenocarcinoma of ovary that could be investigated in future new clinical trials. In addition, the presence of KRAS mutations was associated with better patient outcome.

\section{IGCS19-0478}

\section{A CASE STUDY ON OVARY METASTATIC MELANOMA}

M Rosetti*, A Larre, M Forguieri, V Sartorelli, A Monte, F Coelho. Instituto Brasileiro de Controle ao Cancer, gynecologic oncology, São Paulo, Brazil

\subsection{6/ijgc-2019-IGCS.325}

Objectives Melanoma accounts for 3\% of skin neoplasms in Brazil, with an incidence of $1.7 \%$ in women in the year 2018, according to the National Institute of Cancer. It can appear in any part of the body, but the skin is the primary site in $90 \%$ of cases. In $2-3 \%$ of the cases, the primary site is unknown. In this context, the objective is to present a rare case of ovary metastatic melanoma.

Methods We present a case report of a 36-year-old patient who underwent bilateral oophoroplasty and tubo-ovarian abscess drainage due to an acute inflammatory abdomen. Initially anatomopathological results of ovary dysgerminoma showed a $11 \mathrm{~cm}$ tumor with approximately 10\% tumor necrosis and index mitotic 6/10. Histopathology study showed a superficial spreading melanoma, while immunohistochemistry discrimination was positive for S100, HMB-45, and Melan-A.

Results The case evolved with right inguinal lesion and bilateral adnexal tumors. Positron emission tomography showed multiple peritoneal implants, metastatic lesions in the lumen 